\title{
Dynamics of zonal flows: failure of wave-kinetic theory, and new geometrical optics approximations
}

\author{
Jeffrey B. Parker $\dagger$ \\ Stanford Law School, Stanford, CA 94305, USA \\ (Received 21 April 2016; revised 21 October 2016; accepted 21 October 2016)
}

The self-organisation of turbulence into regular zonal flows can be fruitfully investigated with quasi-linear methods and statistical descriptions. A wave-kinetic equation that assumes asymptotically large-scale zonal flows leads to ultraviolet divergence. From an exact description of quasi-linear dynamics emerges two better geometrical optics approximations. These involve not only the mean flow shear but also the second and third derivative of the mean flow. One approximation takes the form of a new wave-kinetic equation, but is only valid when the zonal flow is quasi-static and wave action is conserved.

Key words: plasma instabilities, plasma nonlinear phenomena, plasma waves

\section{Introduction}

Self-organisation and emergent phenomena in systems with many degrees of freedom and that are far from thermal equilibrium remain a frontier in physics. In fluid dynamics, where turbulence and strong nonlinearities are ubiquitous, the difficulty is amplified because many standard techniques are unavailable.

A striking example of self-organisation is zonal flow, which refers to banded flows alternating in space and quasi-stationary in time. Zonal flow is formed from and coexists with turbulence in the diverse physical contexts of magnetically confined toroidal plasmas (Diamond et al. 2005; Fujisawa 2009; Hillesheim et al. 2016), planetary atmospheres (Vasavada \& Showman 2005) and possibly astrophysical discs (Johansen, Youdin \& Klahr 2009; Kunz \& Lesur 2013). Common to each of these physical systems are directions of symmetry, driven turbulent flow and a gradient in the rotation, density, pressure or other background quantity.

In recent years, a quasi-linear approximation has facilitated progress in the fundamental understanding of zonal flows (Farrell \& Ioannou 2003, 2007; Marston, Conover \& Schneider 2008; Srinivasan \& Young 2012; Bakas \& Ioannou 2013; Parker \& Krommes 2013, 2014; Tobias \& Marston 2013; Constantinou, Farrell \& Ioannou 2014; Bakas, Constantinou \& Ioannou 2015; Constantinou, Farrell \& Ioannou 2016). The quasi-linear approximation involves neglecting the fluctuation self-nonlinearity while retaining the basic nonlinear coupling between mean flows and fluctuations.

† Email address for correspondence: parker68@1lnl.gov 
This truncation eliminates the Kolmogorov cascades and wave-wave interactions, but numerical simulations of quasi-linear dynamics show that fluctuations can still drive the spontaneous formation of zonal flows (Srinivasan \& Young 2012). Hence, even though many fluid systems are naturally turbulent, turbulence per se is not a critical factor in driving zonal flows, and many aspects of the problem can be qualitatively understood in the simpler quasi-linear setting. This approach has found use beyond zonal flows for understanding generation of other large-scale structures such as the magnetorotational dynamo (Squire \& Bhattacharjee 2015) and rolls and streaks in wall-bounded shear flow (Farrell \& Ioannou 2012). In these quasi-linear models, fluctuations are typically driven by external white noise forcing, an analytically convenient assumption. It should be emphasized that the quasi-linear approximation is a model useful for gaining insight, and one does not expect its quantitative predictions to be correct, particularly when the mean flow is weak.

Because the quasi-linear system couples the fluctuations with the mean field, the equations are stochastically linear, despite being dynamically nonlinear. One can therefore apply an averaging procedure and derive an equation of motion for the covariance without facing the standard closure problem of turbulence in which unknown triple correlations appear.

This averaging leads to a coupled set of equations for the two-point, one-time covariance of the fluctuations and the mean flow (Farrell \& Ioannou 2003, 2007; Marston et al. 2008; Srinivasan \& Young 2012). These equations are called CE2, short for second-order cumulant expansion. CE2 offers a set of nonlinear deterministic equations in which rapid fluctuations have been averaged away yet is still equivalent to the quasi-linear dynamics, and is the simplest consistent statistical formulation in which to study inhomogeneous flows.

It is impossible to overstate the importance of CE2 in the context of the quasi-linear model. If statistical inhomogeneity exists in only one dimension, then in a particular limit and with a particular averaging procedure, CE2 describes the exact dynamics of a single realization, not merely the statistics of an ensemble of trajectories (Srinivasan \& Young 2012; Parker \& Krommes 2013). Some questions about the interpretation and relation of statistical ensembles to the actual dynamics of interest can therefore be avoided. This limit is obtained when the domain size in the zonal direction is sufficiently large so that a zonal average becomes equivalent to an ensemble average over the noise, a particular form of ergodicity akin to a thermodynamic limit. No assumption of separation of time or spatial scales is necessary.

With CE2, a basic theoretical framework of zonal flows has been uncovered (Farrell \& Ioannou 2003, 2007; Srinivasan \& Young 2012; Parker \& Krommes 2013, 2014). A statistically homogeneous equilibrium consisting of fluctuations without zonal flows always exists, though it may be unstable. If the incoherent fluctuations are sufficiently intense, they can drive a symmetry-breaking instability that grows into zonal flow. This instability is known as the zonostrophic instability and provides an emergent length and time scale for zonal flows. The instability also has a real eigenvalue, i.e. a perturbation is stationary as it grows.

The zonostrophic instability has been shown to be a generalization of what is sometimes called a modulational, parametric or secondary instability of a primary eigenmode. One tractable calculation examines the stability of a monochromatic wave, e.g. $\psi \sim \mathrm{e}^{\mathrm{i}(\boldsymbol{k} \cdot \boldsymbol{x}-\omega t)}+$ (complex conjugate), to a zonal flow perturbation with wavevector $\boldsymbol{q}$, along with two sidebands $\boldsymbol{k} \pm \boldsymbol{q}$ (Lorenz 1972; Gill 1974; Chen, Lin \& White 2000; Connaughton et al. 2010). A dispersion relation can be calculated; the growth rate of zonal flow depends on the amplitude $|\psi|$ of the pump wave. This dispersion 


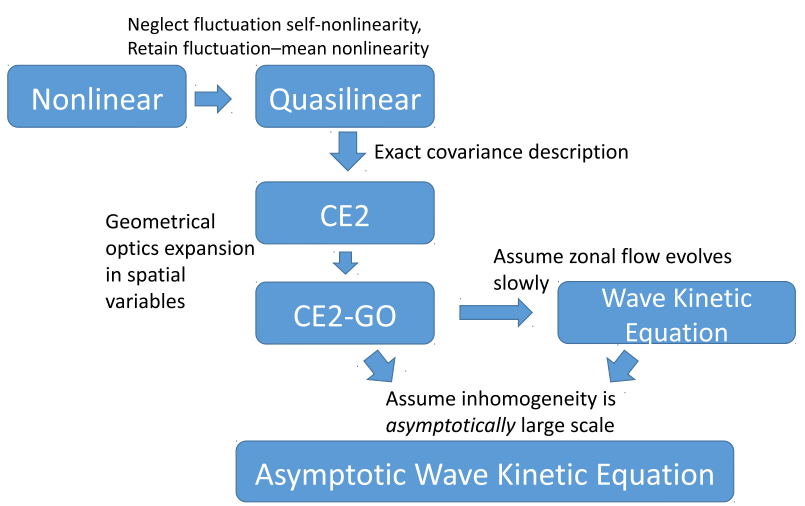

FIGURE 1. Hierarchy of models.

relation can be recovered identically from the CE2 approach by specializing the zonostrophic instability to a monochromatic background spectrum (Parker 2014; Parker \& Krommes 2016). The CE2 approach allows a more general conception of this instability, where the pump can consist not only of a single mode, but also of a full spectrum of incoherent fluctuations.

When zonostrophic instability is marginally unstable, dynamics can be reduced to the real Ginzburg-Landau equation with universal behaviour (Parker \& Krommes 2013, 2014). Qualitative features of the Ginzburg-Landau equation provide insight into behaviour often observed in numerical simulations. For example, merging zonal jets are commonly seen in the transient phase prior to saturation (Huang \& Robinson 1998; Scott \& Polvani 2007). This phenomenon exists within the Ginzburg-Landau equation and can be related to jet stability. As another example, some have remarked about the existence of multiple attractors or dependence on initial conditions (Danilov \& Gurarie 2004; Marcus \& Shetty 2011). This property, too, follows directly from the Ginzburg-Landau equation. More generally, the pattern formation conceptual framework has proven useful (Cross \& Hohenberg 1993; Cross \& Greenside 2009).

All of these results have been understood within the quasi-linear approximation using CE2 in the Charney-Hasegawa-Mima model. While it has not yet been concretely demonstrated that the same structure exists in the original, fully turbulent system, some numerical evidence indicates it does (Parker \& Krommes 2014).

In this paper, we discuss a few approximations to CE2 that offer a simpler, more intuitive form. Two of these invoke wave-kinetic equations, and we explain why this approach breaks down when studying zonal flow dynamics, although it may still prove useful in steady state. We introduce another geometrical optics approximation that may be both accurate and intuitively appealing. The relationship between these models is shown in figure 1.

\section{Quasi-linear and CE2 equations of motion}

The two-dimensional Charney-Hasegawa-Mima equation has been used to model turbulent flow in uniformly magnetized plasma with a density gradient (atmospheric fluid on a rotating planet). We use the generalized Hasegawa-Mima equation (Krommes \& Kim 2000; Smolyakov, Diamond \& Malkov 2000a),

$$
\partial_{t} \zeta(x, y, t)+\boldsymbol{v} \cdot \nabla \zeta-\kappa \partial_{y} \psi=f+D,
$$


where $\zeta$ is the vorticity, $\psi$ is the electric potential (streamfunction) and $\boldsymbol{v}=\hat{z} \times \nabla \psi$ is the fluid velocity. The conventional plasma coordinate system is used where $\kappa$ is the local gradient of plasma density (Coriolis parameter) in the $-x$ direction and $y$ is the zonal direction. $f$ and $D$ represent forcing and dissipation. Variables can be decomposed into mean and fluctuating components, e.g. $\zeta=\bar{\zeta}+\widetilde{\zeta}$, where $\bar{\zeta}=L_{y}^{-1} \int_{0}^{L_{y}} \mathrm{~d} y \zeta$ is a zonally averaged quantity and $L_{y}$ is the periodicity length in the $y$ direction. The generalized Hasegawa-Mima equation involves taking $\widetilde{\zeta}=\bar{\nabla}^{2} \widetilde{\psi}=\left(\nabla^{2}-\rho_{s}^{-2}\right) \widetilde{\psi}$ for fluctuations and $\bar{\zeta}=\nabla^{2} \bar{\psi}$ for the mean flow, where $\rho_{s}$ is the plasma sound radius (deformation radius). In plasma coordinates, lengths are normalized to the sound radius and $\rho_{s}$ should be set to 1; the geophysical barotropic vorticity equation is recovered for $\rho_{s}^{-2}=0$.

The quasi-linear system is obtained by, within the equation for the fluctuations, discarding the terms quadratic in fluctuations. One obtains $\partial_{t} \widetilde{\zeta}+\overline{\boldsymbol{v}} \cdot \nabla \widetilde{\zeta}+\widetilde{\boldsymbol{v}} \cdot \nabla \bar{\zeta}-$ $\kappa \partial_{y} \widetilde{\psi}=f+D$ and $\partial_{t} \bar{\zeta}+\overline{\widetilde{\boldsymbol{v}} \cdot \nabla \widetilde{\zeta}}=D$. With no forcing or dissipation, the fluctuation equation may be written more explicitly as $\partial_{t} \widetilde{\zeta}-\left(\kappa+U^{\prime \prime}\right) \partial_{y} \widetilde{\psi}+U \partial_{y} \widetilde{\zeta}=0$. In a WKB approximation where $U$ is assumed to have slower spatial and temporal dependence than $\zeta$, one can consider an ansatz $\zeta \sim \mathrm{e}^{\mathrm{i}(\boldsymbol{k} \cdot \boldsymbol{x}-\omega(\boldsymbol{k}, \boldsymbol{x}) t)}$. This ansatz yields a wave frequency

$$
\omega(\boldsymbol{k}, x)=\frac{k_{y}\left[\kappa+U^{\prime \prime}(x)\right]}{\bar{k}^{2}}+k_{y} U(x),
$$

where $\bar{k}^{2}=k^{2}+\rho_{s}^{-2}$ and $k^{2}=k_{x}^{2}+k_{y}^{2}$. Many studies have ignored the $U^{\prime \prime}$ term. We show the results so obtained have extremely limited applicability and demonstrate the importance of retaining $U^{\prime \prime}$.

We take the forcing to consist of statistically homogeneous white noise with covariance $F$ and dissipation to consist of a constant friction $\mu$. Viscosity is not necessary to regularize the dynamics in the quasi-linear system because there is no turbulent cascade. Direct numerical simulations of both the original system and the quasi-linear approximation exhibit steady zonal flows (Srinivasan \& Young 2012; Parker \& Krommes 2013).

The CE2 equations can be derived directly from the quasi-linear equations of motion. We quote the results (Srinivasan \& Young 2012; Parker \& Krommes 2013):

$$
\begin{gathered}
\partial_{t} W(x, y, \bar{x}, t)+\left(U_{+}-U_{-}\right) \partial_{y} W-\left(U_{+}^{\prime \prime}-U_{-}^{\prime \prime}\right)\left(\bar{\nabla}^{2}+\frac{1}{4} \partial_{\bar{x}}^{2}\right) \partial_{y} \Psi \\
+\left[2 \kappa+\left(U_{+}^{\prime \prime}+U_{-}^{\prime \prime}\right)\right] \partial_{\bar{x}} \partial_{x} \partial_{y} \Psi=F(x, y)-2 \mu W, \\
\partial_{t} U(\bar{x}, t)+\mu U=-\left.\partial_{\bar{x}} \partial_{x} \partial_{y} \Psi(x, y, \bar{x}, t)\right|_{(x, y)=(0,0)},
\end{gathered}
$$

where $U$ is the zonal flow velocity, $U_{ \pm}=U(\bar{x} \pm x / 2, t)$, and $U^{\prime \prime}=\partial_{\bar{x}}^{2} U$. Here, $\bar{x}$ is the inhomogeneity coordinate - if quantities are statistically homogeneous, there is no dependence on $\bar{x}$. $W$ and $\Psi$ are the two-point, one-time covariance of vorticity and streamfunction, respectively, and are related by $W(x, y, \bar{x}, t)=L_{+} L_{-} \Psi(x, y, \bar{x}, t)$, where $L_{ \pm}=\bar{\nabla}^{2} \pm \partial_{x} \partial_{\bar{x}}+(1 / 4) \partial_{\bar{x}}^{2}$. Equivalently, $W$ is the physical-space Wigner function for $\zeta$ and $(2.3 a)$ is the Wigner transport equation that can be alternatively described in terms of formal Weyl symbols (Krommes \& Parker 2016). The right-hand side of $(2.3 b)$ is the mean force due to the divergence of the Reynolds stress. Periodicity is assumed in $\bar{x}$. 


\section{Asymptotic wave-kinetic equation}

A wave-kinetic equation (WKE) takes the form

$$
\partial_{t} \mathcal{N}(\boldsymbol{k}, \boldsymbol{x}, t)+\frac{\partial \omega}{\partial \boldsymbol{k}} \cdot \frac{\partial \mathcal{N}}{\partial \boldsymbol{x}}-\frac{\partial \omega}{\partial \boldsymbol{x}} \cdot \frac{\partial \mathcal{N}}{\partial \boldsymbol{k}}=S,
$$

where $\mathcal{N}$ is the wave action density and $S$ represents sources and sinks (Connaughton, Nazarenko \& Quinn 2015; Krommes \& Parker 2016). With $S=0$, it is Hamiltonian with $\omega$ generating the equations for wavepacket trajectories through phase space, $\mathrm{d} \boldsymbol{x} / \mathrm{d} t=\nabla_{\boldsymbol{k}} \omega$ and $\mathrm{d} \boldsymbol{k} / \mathrm{d} t=-\nabla_{x} \omega$. For the physical-space coordinates, we write $\boldsymbol{x}=(\bar{x}, \bar{y}) \rightarrow \bar{x}$ because we only allow inhomogeneity in one direction.

A form which has been used as the starting point in many geophysical and plasma physics studies assumes the zonal flows are asymptotically large scale relative to the fluctuations, in which case one neglects $U^{\prime \prime}$ in (2.2) and has $\omega(\boldsymbol{k}, \bar{x})=\kappa k_{y} / \bar{k}^{2}+k_{y} U(\bar{x})$. Equation (3.1) becomes

$$
\partial_{t} \mathcal{N}(\boldsymbol{k}, \bar{x}, t)-k_{x} U^{\prime} \frac{\partial \mathcal{N}}{\partial k_{x}}-\frac{2 \kappa k_{x} k_{y}}{\bar{k}^{4}} \frac{\partial \mathcal{N}}{\partial \bar{x}}=\frac{F(\boldsymbol{k})}{\kappa}-2 \mu \mathcal{N},
$$

where forcing and dissipation have been inserted and the wave action density is $\mathcal{N}=W / \kappa$.

We denote this system as the asymptotic WKE (Manin \& Nazarenko 1994; Smolyakov, Diamond \& Shevchenko 2000b), which we shall see shortly is the limit of the CE2 equations in which the wavelength of zonal flow is taken to be asymptotically large. Justifications for the long-wavelength assumption are tenuous in magnetically confined plasmas, in which the characteristic size of zonal flows is often measured to be comparable to that of the turbulence (Fujisawa et al. 2004; Gupta et al. 2006; Hillesheim et al. 2016). Furthermore, despite the use of the asymptotic WKE in the plasma literature over many years, to our knowledge it has never before been validated by comparing nonlinear numerical solutions to a parent model.

Various studies have inserted into the basic wave-kinetic equation a term that represents the effects of eddy-eddy nonlinear interactions. These interactions conserve energy and enstrophy. In a weak turbulence assumption, these effects can be derived in an asymptotic expansion (Nazarenko 2011). Simple analytic studies sometimes use an ad hoc nonlinear damping term such as $-\mathcal{N}^{2}$, which balances linear instabilities that arise in various plasma models (Diamond et al. 2005). While retaining the effect of eddy-eddy nonlinear interactions in some form is important for many situations, it is unnecessary here. In the quasi-linear approximation, the eddy-eddy nonlinear interactions are absent, which is why an exact statistical theory could be formulated without a closure problem. The lack of eddy-eddy nonlinear interactions makes the quasi-linear system a useful testbed for various statistical theories and approximations of the drift wave-zonal flow problem. If a model is unable to faithfully reproduce the quasi-linear dynamics where these effects are absent, then one has no confidence in that model in the more realistic situation when these effects are included.

Dynamics in the asymptotic WKE is closed by adding the equation for the zonal flow,

$$
\partial_{t} U(\bar{x}, t)+\mu U=\partial_{\bar{x}} \int \frac{\mathrm{d} \boldsymbol{k}}{(2 \pi)^{2}} \frac{\kappa k_{x} k_{y}}{\bar{k}^{4}} \mathcal{N}(\boldsymbol{k}, \bar{x}, t),
$$

where we have used the Fourier transform convention $f(k)=\int \mathrm{d} x \mathrm{e}^{-\mathrm{i} k x} f(x)$. With no forcing or dissipation, it conserves enstrophy within the fluctuations only and conserves total energy of the fluctuations and zonal flow. 


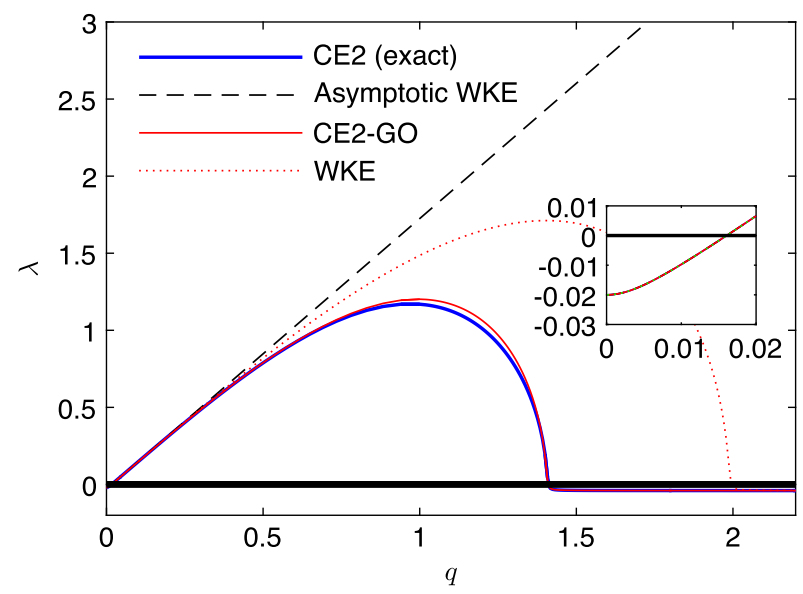

FIGURE 2. Dispersion relation of zonostrophic instability describing linear stage of growth of zonal flows about a homogeneous equilibrium. Inset: zoomed in at small $q$, where all the curves overlap. Parameters: $\mu=0.02, \beta=1, \rho_{s}^{-2}=1, F=4 \pi \varepsilon k_{f} \delta\left(k-k_{f}\right), \varepsilon=1, k_{f}=1$.

The asymptotic WKE is also poorly behaved at small scales. It drives growth of arbitrarily small-scale zonal flow in finite time, violating its fundamental assumption of scale separation as well as precluding any kind of well-behaved solution. The dispersion relation of zonostrophic instability for perturbations $\sim \mathrm{e}^{\lambda t} \mathrm{e}^{\mathrm{i} q \bar{x}}$ is shown in figure 2.

The dispersion relation plotted in figure 2 is calculated as follows. Equation (3.2) contains a homogeneous equilibrium, independent of $\bar{x}$, at $\mathcal{N}_{H}=F / 2 \kappa \mu, U=0$. One writes $\mathcal{N}=\mathcal{N}_{H}+\mathrm{e}^{\mathrm{i} q \bar{x}} \mathrm{e}^{\lambda t} \mathcal{N}_{1}\left(k_{x}, k_{y}\right), U=\mathrm{e}^{\mathrm{i} q \bar{x}} \mathrm{e}^{\lambda t} U_{1}$ and linearises. The linearised form of (3.2a) can be solved for $\mathcal{N}_{1}$ in terms of $U_{1}$, and then substituted into (3.2b), yielding a single nonlinear equation for the eigenvalue $\lambda$ :

$$
\lambda+\mu=-q^{2} \int \frac{\mathrm{d} \boldsymbol{k}}{(2 \pi)^{2}} \frac{k_{x} k_{y}^{2}}{(\lambda+2 \mu) \bar{k}^{4}-2 \mathrm{i} \kappa q k_{x} k_{y}} \kappa \frac{\partial \mathcal{N}_{H}}{\partial k_{x}} .
$$

When $q$ is large, the unstable mode has $\lambda \sim q$. Another solution exists where $\lambda$ is independent of $q$ at large $q$, but this mode is typically damped and is of lesser physical interest. For more details, see appendix A and the supplementary material available at https://doi.org/10.1017/S0022377816001021.

Nonlinear simulations of the asymptotic WKE confirm the ultraviolet divergence. Figure 3 shows the spectrum of the zonal flow in the saturated, steady state for different values of the highest resolved wavenumber $q$. No matter the resolution, the highest modes grow fastest and zonal flow energy concentrates in the highest resolved wavenumbers. For comparison, the figure also shows the same plot for a direct numerical simulation of the quasi-linear system, in which the scale of the zonal flow is not dependent on the numerical resolution. These simulations are pseudospectral, dealiased and nonlinearly conserve energy and enstrophy to machine precision.

For very small $q$, this model correctly predicts for zonostrophic instability that fluctuations provide an effective forcing proportional to $q^{2}$, a regime sometimes described in the literature. Figure 2 shows that at least for some parameters, the $q^{2}$ regime only exists for extremely small $q$. 


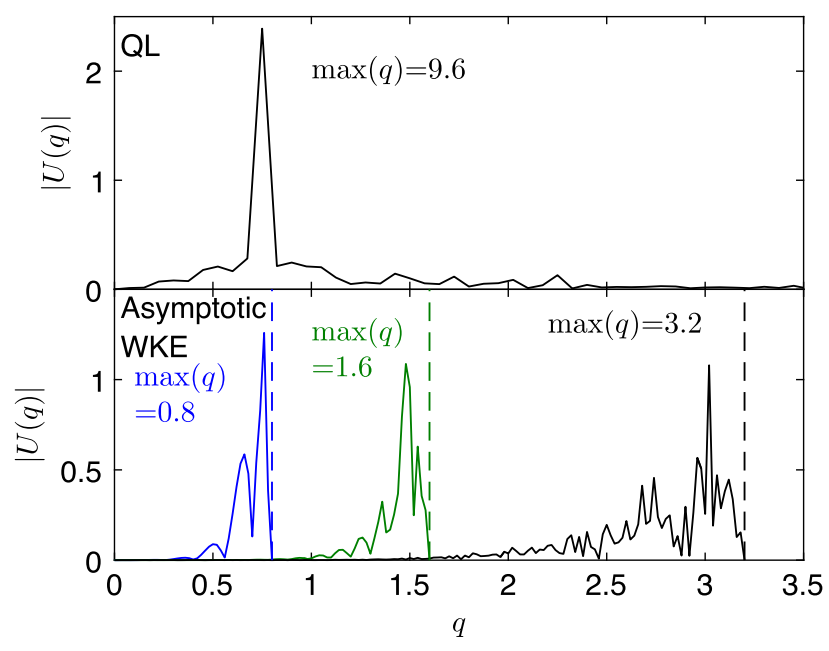

FIGURE 3. Spectrum of the zonal flow $U(q)$ (the Fourier transform of $U(\bar{x})$ ) in the nonlinear saturated state at $t_{f}=6 / \mu$ in simulations of the quasi-linear system and the asymptotic WKE. In each simulation, the zonal flows reach a steady state. For the asymptotic WKE, the results from three simulations are shown, with three different values for the maximum resolved wavenumber of the zonal flow. In each case, the zonal flow energy concentrates at the highest resolved wavenumbers. Same parameters as in figure 2 .

Early studies with the asymptotic WKE correctly deduced that the symmetrybreaking instability is likely a generic phenomenon, which was a key insight (Manin \& Nazarenko 1994; Lebedev et al. 1995; Krommes \& Kim 2000; Smolyakov et al. $2000 b$ ). The asymptotic WKE has been used to predict growth rates of zonal flow in various systems (Diamond et al. 1998; Malkov, Diamond \& Smolyakov 2001b; Anderson et al. 2002, 2006; Wang \& Hahm 2009). As the above results indicate, predicted growth rates will be accurate only at small $q$. Significant error occurs at moderate $q$, and crucially, the asymptotic WKE is completely unable to predict the wavenumber $q$ with maximum growth rate. Aside from growth rates, others have attempted to use the asymptotic WKE to seek nonlinear solutions of zonal flow-turbulence interaction, but due to the system's tendency to dynamically generate small-scale flows, that is invalid from the start (Smolyakov et al. 2000a; Malkov \& Diamond 2001; Malkov, Diamond \& Rosenbluth 2001a; Kaw, Singh \& Diamond 2002; Balescu 2003; Itoh et al. 2004, 2005; Trines et al. 2005; Singh et al. 2014). It appears only one of these studies (Trines et al. 2005) performs numerical simulations of the asymptotic WKE.

Approximations of the asymptotic WKE are perilous because they can elide the growth of small scales present in the model. Sometimes, a random-walk argument based on random zonal flows has been invoked to transform (3.2a) into a diffusion equation (Diamond et al. 1998; Malkov \& Diamond 2001). However, such an assumption stands in contrast with the typically real growth rate of zonal flows and the observations from numerical simulations of Hasegawa-Mima or Hasegawa-Wakatani models that exhibit steady, not random, zonal flows (Numata, Ball \& Dewar 2007; Parker \& Krommes 2013).

\section{Geometrical optics approximations}

We report two reductions of CE2 involving a geometrical optics approximation. 
The first approximation, which we call CE2-GO, involves an expansion in space that is justified when inhomogeneities are weak. The $U_{ \pm}$terms in $(2.3 a)$ are Taylor expanded for small $x$, assuming that the covariance $W$ is small when $x$ is not small. Additionally, only lowest order in $\partial_{\bar{x}}$ is kept, e.g. the lowest-order relation $W(x, y, \bar{x})=$ $\bar{\nabla}^{4} \Psi(x, y, \bar{x})$ is used. After Fourier transforming $(x, y) \rightarrow \boldsymbol{k}$, we obtain

$$
\begin{gathered}
\partial_{t} W(\boldsymbol{k}, \bar{x}, t)-k_{y} U^{\prime} \frac{\partial W}{\partial k_{x}}-k_{y} U^{\prime \prime \prime} \frac{\partial}{\partial k_{x}}\left(\frac{W}{\bar{k}^{2}}\right)-2\left(\kappa+U^{\prime \prime}\right) \frac{k_{x} k_{y}}{\bar{k}^{4}} \frac{\partial W}{\partial \bar{x}}=F(\boldsymbol{k})-2 \mu W, \\
\partial_{t} U(\bar{x}, t)+\mu U=\partial_{\bar{x}} \int \frac{\mathrm{d} \boldsymbol{k}}{(2 \pi)^{2}} \frac{k_{x} k_{y}}{\bar{k}^{4}} W .
\end{gathered}
$$

The dispersion relation for zonally symmetric perturbations about a homogeneous equilibrium can be obtained in a similar manner as (3.3). The result is

$$
\lambda+\mu=-q^{2} \int \frac{\mathrm{d} \boldsymbol{k}}{(2 \pi)^{2}} \frac{k_{x} k_{y}^{2}}{(\lambda+2 \mu) \bar{k}^{4}-2 \mathrm{i} \kappa q k_{x} k_{y}} \frac{\partial}{\partial k_{x}}\left[\left(1-\frac{q^{2}}{\bar{k}^{2}}\right) W_{H}\right],
$$

where $W_{H}=F / 2 \mu$. This dispersion relation is identical to (3.3) except for the factor of $\left(1-q^{2} / \bar{k}^{2}\right)$. This factor, which arises from the $U^{\prime \prime \prime}$ term in $(4.1 a)$, provides the crucial cutoff at large $q$.

In the second approximation, the zonal flow is assumed quasi-static, leading to conservation of wave action density $\mathcal{N}=W /\left(\kappa+U^{\prime \prime}\right)$. A wave-kinetic equation in the form of $(3.1)$ is derived from $(4.1 a)$ by dividing by $\left(\kappa+U^{\prime \prime}\right)$. One obtains

$$
\begin{gathered}
\partial_{t} \mathcal{N}(\boldsymbol{k}, \bar{x}, t)-k_{y}\left(U^{\prime}+\frac{U^{\prime \prime \prime}}{\bar{k}^{2}}\right) \frac{\partial \mathcal{N}}{\partial k_{x}}-2\left(\kappa+U^{\prime \prime}\right) \frac{k_{x} k_{y}}{\bar{k}^{4}} \frac{\partial \mathcal{N}}{\partial \bar{x}}=\frac{F(\boldsymbol{k})}{\kappa+U^{\prime \prime}}-2 \mu \mathcal{N}, \\
\partial_{t} U(\bar{x}, t)+\mu U=\partial_{\bar{x}} \int \frac{\mathrm{d} \boldsymbol{k}}{(2 \pi)^{2}} \frac{k_{x} k_{y}}{\bar{k}^{4}}\left(\kappa+U^{\prime \prime}\right) \mathcal{N}
\end{gathered}
$$

We denote this coupled set as the WKE (Wordsworth 2009). Equation (4.3a) arises from the wave frequency of (2.2). This WKE is invalid when $U^{\prime \prime}$ is rapidly evolving such as during an instability because wave action is not conserved. This formulation therefore may not be used to study the dynamics in which a zonal flow evolves to a steady state. However, in the quasi-steady-state limit, the WKE is valid and equivalent to CE2-GO. The WKE exhibits a close connection to the Rayleigh-Kuo stability criterion, which states that a necessary condition for instability of fixed mean flow is that $\kappa+U^{\prime \prime}$ has a zero somewhere (Kuo 1949); such a condition would create singularities in $\mathcal{N}=W /\left(\kappa+U^{\prime \prime}\right)$.

Both CE2-GO and the WKE nonlinearly conserve enstrophy and energy between the fluctuations and zonal flow, although for the WKE one again must assume quasistatic zonal flow. Within the geometrical optics approximation, the energy density of the fluctuations is $\left(2 L_{x}\right)^{-1} \int_{0}^{L_{x}} \mathrm{~d} \bar{x} \int \mathrm{d} \boldsymbol{k}(2 \pi)^{-2} W(\boldsymbol{k}, \bar{x}) / \bar{k}^{2}$, and the enstrophy density is the same integral with $W$ instead of $W / \bar{k}^{2}$, where $L_{x}$ is the periodicity length in the $x$ direction. The energy density of the zonal flow is $\left(2 L_{x}\right)^{-1} \int_{0}^{L_{x}} \mathrm{~d} \bar{x} U(\bar{x})^{2}$ and the enstrophy density of the zonal flow is $\left(2 L_{x}\right)^{-1} \int_{0}^{L_{x}} \mathrm{~d} \bar{x} U^{\prime}(\bar{x})^{2}$. In the WKE, total wave action is conserved within the fluctuations under steady zonal flow and in the absence of forcing and dissipation.

In the limit of asymptotically large-scale zonal flows in which $U^{\prime \prime}$ and $U^{\prime \prime \prime}$ are neglected, both CE2-GO and the WKE reduce to the asymptotic WKE. 
The dispersion relations for zonostrophic instability in CE2-GO and the WKE are shown in figure 2. CE2-GO agrees remarkably well with the exact CE2, even at $q$ where a scale separation is not satisfied. The $U^{\prime \prime \prime}$ term in (4.1a) and (4.3a) is essential; when only the flow shear $U^{\prime}$ is retained, the dispersion relation grows linearly in $q$. The WKE does not correctly predict the dispersion relation because its fundamental assumption of quasi-static zonal flow is violated.

We emphasize that CE2 is exact for quasi-linear dynamics while CE2-GO is approximate, so CE2-GO should not be preferred just because of its simpler and more intuitive form. Numerical solutions of CE2 are feasible and many studies have been carried out successfully (Farrell \& Ioannou 2003, 2007; Marston et al. 2008; Tobias, Dagon \& Marston 2011; Parker \& Krommes 2013; Constantinou et al. 2014; Parker \& Krommes 2014; Squire \& Bhattacharjee 2015). Moreover, numerically solving CE2 as compared to CE2-GO takes comparable effort as both are three dimensional, although the geometrical optics approximation may reduce the computational cost. Certain analytic calculations are also possible for CE2 (Srinivasan \& Young 2012; Bakas \& Ioannou 2013; Parker \& Krommes 2013, 2014; Bakas et al. 2015).

The utility of CE2-GO and the WKE, if they prove to be accurate, will come from their transparent phase-space formulation, more intuitive interpretation of wave dynamics, and greater possibility of analytic manipulation as compared to CE2. The WKE is valid for studying steady states, and we anticipate it will provide new insight into the behaviour of wavepackets in a steady mean flow.

\section{Discussion}

The asymptotic wave-kinetic equation, where zonal flows are assumed to be asymptotically large scale compared to the fluctuations, violates its own fundamental assumption in finite time. Within this model, the instability that drives zonal flows grows linearly in wavenumber without bound. Nonlinear numerical solutions confirm that zonal flow energy concentrates in the highest resolved wavenumbers. From one point of view, this ultraviolet divergence occurs because the asymptotic WKE attempts to describe the effect of mean flow on the fluctuations solely in terms of the local mean flow shear. Although the asymptotic WKE may remain useful as one of the simplest possible methods for investigating the linear stage of zonal flow growth, the CE2 and CE2-GO models offer more accurate yet still practicable methods that supersede it.

CE2 is an exact description of quasi-linear dynamics, and we have described two geometrical optics approximations that offer simpler and more intuitive phase-space equations. These formulations involve not only the local mean flow shear, but also the second and third derivative of the mean flow. The first formulation, CE2-GO, requires only a spatial scale expansion and agrees with the exact zonostrophic instability dispersion relation remarkably well. The second formulation, a wave-kinetic equation written in terms of conservation of wave action, is valid only when the zonal flow is slowly varying. This requirement precludes the use of the wave-kinetic equation for studying the important dynamics that determines how zonal flows grow and saturate, during which wave action is not conserved. Future work will continue to study the geometrical optics approximations and their validity.

While the quasi-linear approximation provides deep insight into the behaviour of zonal flows, it is important to understand when and why the approximation breaks down. For instance, Kelvin-Helmholtz-like instabilities of shear flow may limit their strength, and the quasi-linear approximation may not capture physics that depends on 
vortex roll up (Connaughton et al. 2010). Comparisons of the quasi-linear system with higher fidelity models offers an approach of further developing our understanding of these kinds of effects. Initial efforts along these lines have been completed, but more remains to be understood (Tobias \& Marston 2013; Constantinou et al. 2016; Marston, Chini \& Tobias 2016)

Finally, although we have emphasized the quasi-linear approximation as a complete nonlinear system that can be analysed in detail, the geometrical optics approximation discussed here is useful more broadly. If one retained the effect of eddy-eddy nonlinearities, the geometrical optics approach may still be used to simplify the exact mean flow-fluctuation interactions.

\section{Acknowledgements}

Useful discussions with N. Constantinou, I. Dodin, J. Krommes, and D. Ruiz are acknowledged. The author is grateful for the helpful comments of an anonymous referee.

\section{Supplementary material}

Supplementary material is available at https://doi.org/10.1017/S0022377816001021.

\section{Appendix A. Numerical calculation of dispersion relation}

The dispersion relation for the zonostrophic instability in the asymptotic WKE, equation (3.3), and in CE2-GO, equation (4.2), can be solved numerically for the eigenvalue $\lambda$ once the parameters $\beta, \mu, q$ and the forcing spectrum $F(\boldsymbol{k})$ are specified. In this appendix we explain the numerical calculation for $\lambda$ shown in figure 2 . We use the asymptotic WKE as an example; the calculation for the other models is analogous. Most choices of forcing $F$ that result in an unstable perturbation $\operatorname{Re}(\lambda)>0$ have a purely real $\lambda$.

For the forcing $F$, we take a thin-ring isotropic forcing $F(k)=4 \pi \varepsilon k_{f} \delta\left(k-k_{f}\right)$, a convenient simplification. If $\rho_{s}^{-2}=0$, then $\varepsilon$ is equal to the energy input by the forcing. After integrating by parts in $k_{x}$, switching to polar coordinates $k_{x}=k \cos \phi$ and $k_{y}=$ $k \sin \phi$, and integrating over the delta function, the dispersion relation becomes

$$
\lambda+\mu=q^{2} \frac{\varepsilon}{\mu} k_{f}^{4}(\lambda+2 \mu)\left(1+m_{f}\right) \int_{0}^{2 \pi} \frac{\mathrm{d} \phi}{2 \pi} \frac{\left(1+m_{f}-4 \cos ^{2} \phi\right) \sin ^{2} \phi}{\left[(\lambda+2 \mu) k_{f}^{2}\left(1+m_{f}\right)^{2}-2 \mathrm{i} \kappa q \cos \phi \sin \phi\right]^{2}},
$$

where $m_{f}=k_{f}^{-2} \rho_{s}^{-2}$. Assuming real $\lambda$, one can rationalize the integrand and discard the imaginary part because it vanishes upon integration, and then proceed using robust one-dimensional numerical root finders.

Python code to carry out the calculations and reproduce some of the curves in figure 2 is available in the supplementary material.

\section{REFERENCES}

Anderson, J., Nordman, H., Singh, R. \& Weiland, J. 2002 Zonal flow generation in ion temperature gradient mode turbulence. Phys. Plasmas 9 (11), 4500-4506.

Anderson, J., Nordman, H., Singh, R. \& Weiland, J. 2006 Zonal flow generation in collisionless trapped electron mode turbulence. Plasma Phys. Control. Fusion 48 (5), 651-661.

Bakas, N. A., Constantinou, N. C. \& Ionnnou, P. J. 2015 S3t stability of the homogeneous state of barotropic beta-plane turbulence. J. Atmos. Sci. 72 (5), 1689-1712. 
BAKas, N. A. \& IOANnou, P. J. 2013 On the mechanism underlying the spontaneous emergence of barotropic zonal jets. J. Atmos. Sci. 70, 2251-2271.

BAlescu, R. 2003 Drift-wave turbulence and zonal flow generation. Phys. Rev. E 68, 046409.

Chen, L., Lin, Z. \& White, R. 2000 Excitation of zonal flow by drift waves in toroidal plasmas. Phys. Plasmas 7 (8), 3129-3132.

Connaughton, C., Nazarenko, S. \& Quinn, B. 2015 Rossby and drift wave turbulence and zonal flows: the Charney-Hasegawa-Mima model and its extensions. Phys. Rep. 604, 1-71; Rossby and drift wave turbulence and zonal flows: the Charney-Hasegawa-Mima model and its extensions.

Connaughton, C. P., Nadiga, B. T., Nazarenko, S. V. \& Quinn, B. E. 2010 Modulational instability of Rossby and drift waves and generation of zonal jets. J. Fluid Mech. 654, 207-231.

Constantinou, N. C., Farrell, B. F. \& Ioannou, P. J. 2014 Emergence and equilibration of jets in beta-plane turbulence: applications of stochastic structural stability theory. J. Atmos. Sci. 71 (5), 1818-1842.

Constantinou, N. C., Farrell, B. F. \& Ioannou, P. J. 2016 Statistical state dynamics of jet-wave coexistence in barotropic beta-plane turbulence. J. Atmos. Sci. 73 (5), 2229-2253.

Cross, M. \& Greenside, H. 2009 Pattern Formation and Dynamics in Nonequilibrium Systems. Cambridge University Press.

Cross, M. C. \& Hohenberg, P. C. 1993 Pattern formation outside of equilibrium. Rev. Mod. Phys. 65, 851-1112.

Danilov, S. \& Gurarie, D. 2004 Scaling, spectra and zonal jets in beta-plane turbulence. Phys. Fluids 16 (7), 2592-2603.

Diamond, P. H., Itoh, S.-I., Iтон, K. \& Hahm, T. S. 2005 Zonal flows in plasma - a review. Plasma Phys. Control. Fusion 47 (5), R35-R161.

Diamond, P. H., Rosenbluth, M. N., Hinton, F. L., Malkov, M., Fleischer, J. \& SMOLYAKov, A. 1998 Dynamics of zonal flows and self-reguating drift-wave turbulence. In Proceedings of the 17th IAEA Fusion Energy Conference. TH3/1.

Farrell, B. F. \& IoAnnou, P. J. 2003 Structural stability of turbulent jets. J. Atmos. Sci. 60 (17), 2101-2118.

FARrell, B. F. \& IOANnou, P. J. 2007 Structure and spacing of jets in barotropic turbulence. J. Atmos. Sci. 64 (10), 3652-3665.

FARrell, B. F. \& IOANnOU, P. J. 2012 Dynamics of streamwise rolls and streaks in turbulent wall-bounded shear flow. J. Fluid Mech. 708, 149-196.

Fujisawa, A. 2009 A review of zonal flow experiments. Nucl. Fusion 49 (1), 013001.

Fujisawa, A., Itoh, K., Iguchi, H., Matsuoka, K., OKamura, S., Shimizu, A., Minami, T., Yoshimura, Y., NagaOKa, K., TAKahashi, C. et al. 2004 Identification of zonal flows in a toroidal plasma. Phys. Rev. Lett. 93, 165002.

GILl, A. 1974 The stability of planetary waves on an infinite beta-plane. Geophys. Fluid Dyn. 6, 29-47.

Gupta, D. K., Fonck, R. J., McKee, G. R., Schlossberg, D. J. \& Shafer, M. W. 2006 Detection of zero-mean-frequency zonal flows in the core of a high-temperature tokamak plasma. Phys. Rev. Lett. 97, 125002.

Hillesheim, J. C., Delabie, E., Meyer, H., Maggi, C. F., Meneses, L., Poli, E. \& CONTRIBUTORS, J. 2016 Stationary zonal flows during the formation of the edge transport barrier in the jet tokamak. Phys. Rev. Lett. 116, 065002.

Huang, H.-P. \& Robinson, W. A. 1998 Two-dimensional turbulence and persistent zonal jets in a global barotropic model. J. Atmos. Sci. 55 (4), 611-632.

Itoh, K., HallatscheK, K., Itoh, S.-I., Diamond, P. H. \& Toda, S. 2005 Coherent structure of zonal flow and onset of turbulent transport. Phys. Plasmas 12, 062303.

Itoh, K., Hallatschek, K., Toda, S., Itoh, S.-I., Diamond, P. H., Yagi, M. \& Sanuki, H. 2004 Collisional effects on coherent structures of zonal flows and turbulent transport. Plasma Phys. Control. Fusion 46 (5A), A335-A340.

Johansen, A., Youdin, A. \& Klahr, H. 2009 Zonal flows and long-lived axisymmetric pressure bumps in magnetorotational turbulence. Astrophys. J. 697 (2), 1269-1289. 
KaW, P., Singh, R. \& Diamond, P. H. 2002 Coherent nonlinear structures of drift wave turbulence modulated by zonal flows. Plasma Phys. Control. Fusion 44 (1), 51-59.

Krommes, J. A. \& Kim, C.-B. 2000 Interactions of disparate scales in drift-wave turbulence. Phys. Rev. E 62, 8508-8539.

Krommes, J. A. \& Parker, J. B. 2016 Statistical closures and zonal flows. In Zonal Jets (ed. B. Galperin \& P. Read). Cambridge University Press, (in press).

KUnZ, M. W. \& Lesur, G. 2013 Magnetic self-organization in Hall-dominated magnetorotational turbulence. Mon. Not. R. Astron. Soc. 434, 2295-2312.

KUo, H.-L. 1949 Dynamic instability of two-dimensional nondivergent flow in a barotropic atmosphere. J. Met. 6 (2), 105-122.

Lebedev, V. B., Diamond, P. H., Shapiro, V. D. \& Soloviev, G. I. 1995 Modulational interaction between drift waves and trapped ion convective cells: a paradigm for the self-consistent interaction of large-scale sheared flows with small-scale fluctuations. Phys. Plasmas 2 (12), 4420-4431.

Lorenz, E. N. 1972 Barotropic instability of Rossby wave motion. J. Atmos. Sci. 29, 258-265.

Malkov, M. A. \& DiAmond, P. H. 2001 Bifurcation and scaling of drift wave turbulence intensity with collisional zonal flow damping. Phys. Plasmas 8 (9), 3996-4009.

Malkov, M. A., Diamond, P. H. \& Rosenbluth, M. N. 2001a On the nature of bursting in transport and turbulence in drift wave-zonal flow systems. Phys. Plasmas 8 (12), 5073-5076.

Malkov, M. A., Diamond, P. H. \& SMolyakov, A. $2001 b$ On the stability of drift wave spectra with respect to zonal flow excitation. Phys. Plasmas 8 (5), 1553-1558.

Manin, D. Y. \& NAZAREnKo, S. V. 1994 Nonlinear interaction of small-scale Rossby waves with an intense large-scale zonal flow. Phys. Fluids 6 (3), 1158-1167.

MARCUS, P. S. \& SHETTY, S. 2011 Jupiter's zonal winds: are they bands of homogenized potential vorticity organized as a monotonic staircase? Phil. Trans. R. Soc. Lond. A 369 (1937), 771-795.

Marston, J. B., Chini, G. P. \& Tobias, S. M. 2016 Generalized quasilinear approximation: application to zonal jets. Phys. Rev. Lett. 116, 214501.

Marston, J. B., Conover, E. \& Schneider, T. 2008 Statistics of an unstable barotropic jet from a cumulant expansion. J. Atmos. Sci. 65 (6), 1955-1966.

NAZARENKo, S. 2011 Wave Turbulence. Springer.

NumatA, R., BALl, R. \& DEWAR, R. L. 2007 Bifurcation in electrostatic resistive drift wave turbulence. Phys. Plasmas 14 (10), 102312.

PARKer, J. B. 2014 Zonal flows and turbulence in fluids and plasmas. PhD thesis, Princeton University.

Parker, J. B. \& Krommes, J. A. 2013 Zonal flow as pattern formation. Phys. Plasmas 20, 100703.

PARKer, J. B. \& Krommes, J. A. 2014 Generation of zonal flows through symmetry breaking of statistical homogeneity. New J. Phys. 16, 035006.

PARKer, J. B. \& Krommes, J. A. 2016 Zonal flow as pattern formation. In Zonal Jets (ed. B. Galperin \& P. Read). Cambridge University Press, arXiv:1503.07498.

ScotT, R. K. \& Polvani, L. M. 2007 Forced-dissipative shallow-water turbulence on the sphere and the atmospheric circulation of the giant planets. J. Atmos. Sci. 64 (9), 3158-3176.

Singh, R., Singh, R., Kaw, P., GÜrCAN, O. D. \& Diamond, P. H. 2014 Coherent structures in ion temperature gradient turbulence-zonal flow. Phys. Plasmas 21, 102306.

Smolyakov, A. I., Diamond, P. H. \& Malkov, M. $2000 a$ Coherent structure phenomena in drift wave-zonal flow turbulence. Phys. Rev. Lett. 84, 491-494.

Smolyakov, A. I., Diamond, P. H. \& Shevchenko, V. I. $2000 b$ Zonal flow generation by parametric instability in magnetized plasmas and geostrophic fluids. Phys. Plasmas 7 (5), 1349-1351.

Squire, J. \& Bhattacharjee, A. 2015 Statistical simulation of the magnetorotational dynamo. Phys. Rev. Lett. 114, 085002.

SRInivasan, K. \& Young, W. R. 2012 Zonostrophic instability. J. Atmos. Sci. 69, 1633-1656.

Tobias, S. M., DAgon, K. \& MARston, J. B. 2011 Astrophysical fluid dynamics via direct statistical simulation. Astrophys. J. 727 (2), 127. 
Tobias, S. M. \& Marston, J. B. 2013 Direct statistical simulation of out-of-equilibrium jets. Phys. Rev. Lett. 110, 104502.

Trines, R., Bingham, R., Silva, L. O., Mendonça, J. T., Shukla, P. K. \& Mori, W. B. 2005 Quasiparticle approach to the modulational instability of drift waves coupling to zonal flows. Phys. Rev. Lett. 94, 165002.

Vasavada, A. R. \& Showman, A. P. 2005 Jovian atmospheric dynamics: an update after Galileo and Cassini. Rep. Prog. Phys. 68, 1935-1996.

WAng, L. \& HAHM, T. S. 2009 Theory of fine-scale zonal flow generation from trapped electron mode turbulence. Phys. Plasmas 16, 082302.

Wordsworth, R. D. 2009 A phase-space study of jet formation in planetary-scale fluids. Phys. Fluids 21 (5), 056602. 